\title{
Efecto de la tormenta geomagnética del 23 de abril de 2012 en el contenido electrónico total de la ionosfera sobre la región mediterránea
}

\author{
MARTA RoDRÍGUEZ-BOUZA ${ }^{1}$, MIGUEL HERRAIZ SARACHAGA ${ }^{1,2}$, \\ GRACIA RODRÍGUEZ-CADEROT ${ }^{3,4}$, SANDRO M. RADICELLA ${ }^{5}$ \\ ${ }^{1}$ Departamento de Física de la Tierra, Astronomía y Astrofísica I (Geofísica y Meteorología), \\ Facultad de Ciencias Físicas, Universidad Complutense de Madrid (UCM), Spain, \\ mrodriguezbouza@ucm.es \\ ${ }^{2}$ Instituto de Geociencias, (UCM, CSIC), Spain \\ ${ }^{3}$ Sec. Dptal. Astronomía y Geodesia, Facultad de Matemáticas, UCM, Spain \\ ${ }^{4}$ Instituto de Matemáticas Interdisciplinar UCM, Spain \\ ${ }^{5}$ Telecommunications/ICT Development Laboratory (T/ICT4D), Abdus Salam International Center for \\ Theoretical Physics (ICTP), Italy
}

Recibido: 20/05/2014

Aceptado: 26/09/2014

\begin{abstract}
Resumen
Las tormentas geomagnéticas son variaciones del campo magnético terrestre de varias horas de duración y de carácter planetario cuyo origen es la llegada a la Tierra de viento solar perturbado, emitido por una eyección de masa coronal, en el que la componente $\mathrm{Z}$ del campo magnético interplanetario tiene orientación hacia el Sur. Una tormenta geomagnética puede dar lugar a una tormenta ionosférica que se caracteriza principalmente por una variación de la densidad electrónica de la capa F2 de la ionosfera. El interés de estas tormentas ionosféricas es su gran influencia en los observables GNSS y, por tanto, su posible efecto en la precisión en el posicionamiento.

Este trabajo describe los fenómenos asociados a la tormenta geomagnética del 23 de abril de 2012. Su origen fue una eyección de masa coronal ocurrida el día 19 que modificó el viento solar produciendo un incremento en su velocidad y cambios en el campo magnético interplanetario. La llegada a la Tierra del viento solar perturbado causó una tormenta geomagnética con inicio el día 23 y que duró hasta el día 27. A su vez, esta tormenta originó una tormenta ionosférica en la región Mediterránea que tuvo varias fases cuyas características dependen de la latitud, la longitud y la etapa de la tormenta geomagnética durante la que se produce.
\end{abstract}

Palabras clave: Tormenta geomagnética, ionosfera, TEC.

Effect of the geomagnetic storm of April 23, 2012, on the ionospheric total electron content over the Mediteranean Region

\begin{abstract}
Geomagnetic storms are disturbances of the terrestrial magnetic field of several hours with a global character. The origin of this disturbance is the arrival of solar wind disturbed by a coronal mass ejection in which the interplanetary magnetic field has a southward $\mathrm{Z}$ component. A geomagnetic storm may result in an ionospheric storm which is mainly characterized by a disturbance of the electron density of the F2-layer of the ionosphere. These ionospheric storms have a major influence on GNSS observables and thus can affect the accuracy in positioning.
\end{abstract}


This work describes the phenomena associated to the geomagnetic storm of 23 of April 2012. The origin was a coronal mass ejection produced the $19^{\text {th }}$ that increased the velocity of the solar wind and changed the interplanetary magnetic field. The arrival of the disturbed solar wind to the Earth triggered a geomagnetic storm that began on $23^{\text {th }}$ and lasted until the $27^{\text {th }}$. This geomagnetic storm caused an ionospheric storm over Mediterranean region which had several phases whose characteristics depend on latitude, longitude and the stage of the geomagnetic storm in which takes place.

Keywords: Geomagnetic storm, ionosphere, TEC.

Sumario: Introducción. 1. Datos y metodología. 2. Sol y viento solar. 3. Tormenta geomagnética. 4. Tormenta ionosférica. 5. Conclusiones. Agradecimientos. Referencias Bibliográficas.

\section{Referencia normalizada}

Rodríguez-Bouza, M., Herraiz, M., Rodríguez-Caderot, G., Radicella, S.M. (2012). Efecto de la tormenta geomagnética del 23 de abril de 2012 en el contenido electrónico total de la ionosfera sobre la región mediterránea. Física de la Tierra, Vol. 26, 101-117.

\section{Introducción}

El comportamiento ionosférico anómalo producido por fenómenos solares extremos reviste un gran interés debido a sus efectos sobre los sistemas tecnológicos, en particular sobre el posicionamiento y la navegación satelital. El estudio de las perturbaciones ionosféricas se ha incrementado en los ultimos años gracias a la creciente disponibilidad de datos obtenidos a partir de redes GNSS permanentes. El tema ha recibido una gran atención en América del Norte donde se han registrado tormentas muy severas, como las ocurridas en noviembre de 2003 (tormentas de Halloween) que originaron fallos en los sistemas de navegación GNSS (Komjathy et al., 2005; Basu et al., 2008). En Europa también se han realizado estudios (Bergeot et al., 2011; Spoogli et al., 2009) que muestran que en esta región se pueden sufrir también los efectos adversos de las tormentas ionosféricas.

Las tormentas ionosféricas son consecuencia de las tormentas geomagnéticas. Estas tormentas, también llamadas magnéticas, son perturbaciones del campo magnético de la Tierra originadas por la llegada al geoespacio de viento solar emitido en un agujero coronal o una eyección de masa coronal, que presenta valores muy elevados en su densidad y velocidad. El viento solar perturbado produce un aumento de la presión sobre la magnetosfera que modifica su estado el hemisferio iluminado, juntando las líneas de campo y aumentando, por tanto, su densidad (Buonsanto, 1999). Además, en esta situación de compresión se produce una intensificación de las corrientes magnetosféricas debido a un aumento del fenómeno de reconexión en el lado nocturno y a una compresión de la magnetocola. Las tormentas geomagnéticas tienen tres fases: inicial, principal y de recuperación (Parkinson, 1983). La fase inicial se debe a la compresión de las líneas de campo de la magnetosfera producida por el aumento de la presión dinámica del viento solar y no se presenta en todas las tormentas geomagnéticas. La fase principal es la etapa en la que se genera una disminución de la componente horizontal del campo 
magnético terrestre debida a la intensificación de la corriente de anillo producida por la entrada de partículas energéticas. La fase de recuperación es la fase de regreso al equilibrio inicial. Para caracterizar las tormentas geomagnéticas se utilizan distintos indicadores de actividad geomagnética. Uno de los más importantes es el índice Dst, Disturbance Storm Time, que se obtiene a partir de la componente $\mathrm{H}$ del campo geomagnético medida en una red de cuatro observatorios geomagnéticos situados cerca del ecuador magnético y con una separación que les permite cubrir todo el perímetro terrestre. Este índice proporciona una medida de la variación del campo magnético debida al anillo de corriente ecuatorial. Durante la fase inicial de la tormenta geomagnética el valor de Dst aumenta levemente, y durante la fase principal disminuye hasta que se inicia la etapa de recuperación. Durante esta fase el valor del índice aumenta poco a poco hasta alcanzar su valor en el equilibrio que se sitúa en torno a 0nT. Otro de los parámetros que cuantifica las tormentas geomagnéticas es el índice $\mathrm{Kp}$, que es un índice planetario derivado del índice $\mathrm{K}$ (indicador que mide la perturbación de la componente horizontal y la declinación en un Observatorio).

El parámetro ionosférico utilizado en este estudio es el TEC que se define como el número de electrones contenido en una columna de un metro cuadrado de sección extendida desde el receptor hasta el satélite. Para describir el contenido de electrones en la ionosfera se definen dos parámetros:

- $\quad$ STEC: contenido oblicuo de electrones. Es el TEC correspondiente a una columna oblicua de un metro cuadrado de sección que se extiende desde el receptor hasta el satélite, es decir:

$$
s T E C=\int_{\text {Receptor }}^{\text {Satélite }} N d s
$$

- vTEC: contenido vertical de electrones. Es el TEC correspondiente a una columna vertical de un metro cuadrado de sección que se extiende desde el receptor hasta el satélite, es decir:

$$
\text { vTEC }=\int_{\text {Receptor }}^{\text {Satélite }} N d h
$$

En estas expresiones $\mathrm{N}$ es la densidad electrónica, ds el elemento diferencial de longitud oblicuo y dh el elemento diferencial de longitud vertical. La unidad del TEC, TECU, vale $10^{16}$ electrones $/ \mathrm{m}^{2}$.

En épocas de alta actividad solar, como los meses de primavera del año 2012, los valores del TEC en el área mediterranea varían entre 5-8 TECus durante la noche y 25-40 TECus en el máximo que se produce a mediodía. Los valores más altos corresponden a la zona Sur de la región analizada y los más bajos a las latitudes en torno a $50^{\circ}$.

Las tormentas ionosféricas se caracterizan por variaciones anómalas de la densidad electrónica de la capa F2 de la ionosfera y por tanto en variaciones del contenido total de electrones, TEC, de la ionosfera (Kelly, 2009). Pueden dividirse 
en fases y, dependiendo de si se produce un incremento o una disminución de la densidad electrónica, la fase se clasificará como positiva o negativa (Huang et al., 2005).

\section{Datos y metodología}

En este trabajo se han utilizado datos de múltiples orígenes para realizar el estudio completo de los fenómenos asociados con la tormenta geomagnética, desde su origen en el Sol hasta su efecto en la ionosfera.

Para analizar el efecto del fenómeno en el viento solar se han utilizado medidas del satélite WIND, en concreto la velocidad del viento solar y la componente vertical del campo magnético interplanetario, z-GSM. Estos datos son proporcionados por el Goddard Space Flight Center a través de su servicio OMNIWeb.

El estudio del efecto de la perturbación sobre el campo magnético terrestre se ha llevado a cabo considerando los índices planetarios Kp y Dst. Además, se han analizado las variaciones del campo magnético en los observatorios de San Pablo de los Montes (España), Duronia (Italia) y Pedeli (Grecia) (Figura 1). Los valores del índice Dst son proporcionados por el World Data Center for Geomagnetism de Kyoto y las variaciones del campo magnético han sido obtenidas de la base de datos de Intermagnet.

Los efectos en la ionosfera se analizan a través del TEC. Para obtener este parámetro se han utilizado archivos RINEX de estaciones GNSS permanentes situadas sobre la región de estudio (Figura 1) de las redes EUREF Permanent Network (EPN) e International GNSS Service, IGS. Estos archivos, junto con los de navegación satelital tanto de la constelación GPS como de la constelación GLONAS, son procesados con un algoritmo (Ciraolo, 2012) que proporciona los valores de vTEC y sTEC cada minuto. La estimación de estos parámetros se basa en el retardo que introduce la ionosfera en la propagación de señales electromagnéticas. Se puede estimar el TEC a partir de la expresión:

$$
T E C=S_{i j t}-\beta_{i}-\gamma_{j}-\lambda
$$

donde los subíndices $\mathrm{i}, \mathrm{j}, \mathrm{t}$ hacen referencia al satélite, receptor y época, respectivamente. Por su parte, $S, \beta$ y $\gamma$ representan los retardos introducidos por la ionosfera y por el hardware del satélite y del receptor, respectivamente. A su vez, $\lambda$ agrupa los errores introducidos por posibles reflexiones de la señal. Es necesario hacer una estimación de los parámetros $\beta_{\mathrm{i}}, \gamma_{\mathrm{j}}$ y $\lambda$ para poder obtener el valor del TEC. Esta estimación se conoce como calibración y es la base del algoritmo. Para su obtención, el TEC se parametriza y linealiza en función de la densidad electrónica, $\mathrm{Ne}$, y la elevación del satélite, $\chi$, considerando una ionosfera formada por capas. Tras esta parametrización se realiza un ajuste por mínimos cuadrados de la ecuación (3) para obtener los valores de $\beta_{\mathrm{i}}, \gamma_{\mathrm{j}}$ y $\lambda$, y con ellos el valor del sTEC y vTEC época por época, así como el valor de la latitud y la longitud del Ionospheric Pierce Point, IPP. El IPP es el punto donde la señal satélite-receptor intersecta con 
la ionosfera considerada como una capa delgada situada a $350 \mathrm{~km}$ de altura donde se encuentran concentrados todos los electrones.

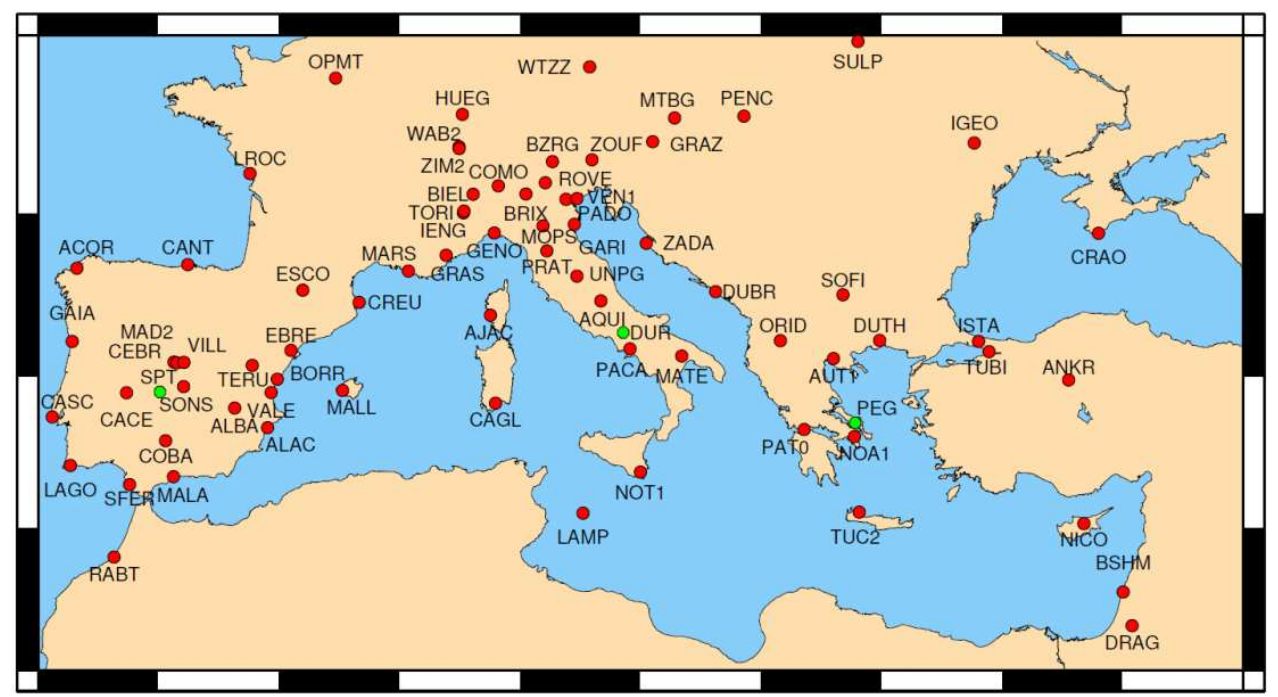

Fig. 1. Estaciones GNSS (rojo) y observatorios de Intermagnet (verde) utilizados en el estudio.

Para detectar las tormentas ionosféricas se ha analizado la variación relativa del vTEC, vTECrel. Este parámetro se obtiene como:

$$
\operatorname{vTECrel}_{i}(\%)=\frac{v \operatorname{TEC}_{i}-v T E C m_{i}}{v T E C m_{i}} \cdot 100
$$

Donde vTECm es el valor medio de vTEC calculado a partir de 20 días geomagnéticamente tranquilos en torno a la tormenta geomagnética estudiada. Se consideran días tranquilos aquellos en los que el índice Dst tiene valores comprendidos entre $\pm 20 \mathrm{nT}$. El subíndice i hace referencia a la época.

La primera parte del estudio ha sido el análisis de los efectos latitudinales de la tormenta en distintas longitudes. Para ello se han seleccionado estaciones en torno a los meridianos $4^{\circ} \mathrm{W}, 0^{\circ} \mathrm{E}, 9^{\circ} \mathrm{E}, 12^{\circ} \mathrm{E}, 16^{\circ} \mathrm{E}, 23^{\circ} \mathrm{E}$ y $34^{\circ} \mathrm{E}$ como se índica en la Tabla 1. Se ha realizado también el mismo análisis para estudiar los efectos longitudinales. Para ello se han seleccionado estaciones en torno a los paralelos $39^{\circ} \mathrm{N}, 44^{\circ} \mathrm{N}$ y $48^{\circ} \mathrm{N}$. En ambos casos se trabaja con coordenadas geográficas. Las estaciones utilizadas para esta parte del estudio se listan en la Tabla 2. En total se han analizado más de 2000 archivos RINEX de 42 estaciones correspondientes a 48 días.

En el análisis de toda la región se ha calculado el vTEC en los IPP. Este parámetro se obtiene con los valores de sTEC, elevación del satélite, latitud y 
longitud del IPP proporcionados por el algoritmo. Los valores del vTEC en los IPP se interpolan con el método Kriging (Webster y Oliver, 2001) para obtener el vTEC en una malla de $1^{\circ} \times 1^{\circ}$. En este parte del estudio se ha trabajado con más de 3700 archivos de todas las estaciones disponibles en la región de estudio.

En todo el análisis, para comparar los datos de los eventos solares, del viento solar, del campo geomagnético y del TEC, se ha utilizado el sistema horario de Tiempo Universal Coordinado (UTC).

Tabla 1. Estaciones utilizadas para el análisis latitudinal.

\begin{tabular}{|c|c|c|c|c|c|c|c|}
\hline \multicolumn{2}{|c|}{ Meridiano $\mathbf{4}^{\mathbf{}} \mathbf{W}$} & \multicolumn{2}{c|}{ Meridiano 0 $^{\mathbf{}} \mathbf{E}$} & \multicolumn{2}{c|}{ Meridiano $\mathbf{9}^{\mathbf{}} \mathbf{E}$} & \multicolumn{2}{c|}{ Meridiano $\mathbf{1 2}^{\mathbf{o}} \mathbf{E}$} \\
\hline Estación & Latitud & Estación & Latitud & Estación & Latitud & Estación & Latitud \\
\hline MALA & $36.73^{\circ}$ & ALAC & $38.34^{\circ}$ & CAGL & $39.14^{\circ}$ & LAMP & $35.50^{\circ}$ \\
\hline COBA & $37.92^{\circ}$ & VALE & $39.45^{\circ}$ & AJAC & $41.93^{\circ}$ & UNPG & $43.12^{\circ}$ \\
\hline SONS & $36.68^{\circ}$ & EBRE & $40.52^{\circ}$ & GENO & $44.12^{\circ}$ & GARI & $44.68^{\circ}$ \\
\hline MAD2 & $40.43^{\circ}$ & ESCO & $42.69^{\circ}$ & COMO & $45.80^{\circ}$ & BZRG & $46.48^{\circ}$ \\
\hline CANT & $43.47^{\circ}$ & \multicolumn{9}{c}{} & WTZZ & $49.14^{\circ}$ \\
\hline
\end{tabular}

\begin{tabular}{|c|c|c|c|c|c|}
\hline \multicolumn{2}{|c|}{ Meridiano $\mathbf{1 6}^{\mathbf{}} \mathbf{E}$} & \multicolumn{2}{c|}{ Meridiano $\mathbf{2 3}^{\mathbf{}} \mathbf{E}$} & \multicolumn{2}{c|}{ Meridiano $\mathbf{3 4}^{\mathbf{o}} \mathbf{E}$} \\
\hline Estación & Latitud & Estación & Latitud & Estación & Latitud \\
\hline MATE & $40.65^{\mathbf{0}}$ & TUC2 & $35.53^{\circ}$ & BSHM & $32.78^{\circ}$ \\
\hline ZADA & $44.11^{\mathbf{o}}$ & NOA1 & $38.05^{\circ}$ & NICO & $35.14^{\mathbf{o}}$ \\
\hline GRAZ & $47.07^{\circ}$ & AUT1 & $40.57^{\circ}$ & ANKR & $39.89^{\circ}$ \\
\hline \multirow{2}{*}{} & SOFI & $42.56^{\circ}$ & CRAO & $44.41^{\mathbf{o}}$ \\
\cline { 2 - 6 } & SULP & $49.84^{\circ}$ & \multicolumn{2}{c}{} \\
\cline { 2 - 5 }
\end{tabular}

Tabla 2. Estaciones utilizadas para el análisis longitudinal.

\begin{tabular}{|c|c|c|c|c|c|}
\hline \multicolumn{2}{|c|}{ Paralelo 39 } & \multicolumn{2}{c|}{ Paralelo $4^{\mathbf{}} \mathbf{N}$} & \multicolumn{2}{c|}{ Paralelo $\mathbf{4 8}^{\mathbf{}} \mathbf{N}$} \\
\hline Estación & Longitud & Estación & Longitud & Estación & Longitud \\
\hline CASC & $-9.42^{\circ}$ & CANT & $-3.80^{\circ}$ & LROC & $-1.122^{\circ}$ \\
\hline SONS & $-3.96^{\circ}$ & ESCO & $0.98^{\circ}$ & OPMT & $2.34^{\circ}$ \\
\hline VALE & $-0.34^{\circ}$ & CREU & $3.32^{\circ}$ & WAB2 & $7.46^{\circ}$ \\
\hline MALL & $2.63^{\circ}$ & IENG & $7.64^{\circ}$ & BZRG & $11.34^{\circ}$ \\
\hline MATE & $16.70^{\circ}$ & MOPS & $10.95^{\circ}$ & GRAZ & $15.49^{\circ}$ \\
\hline PAT0 & $21.79^{\circ}$ & DUBR & $18.11^{\circ}$ & PENC & $19.28^{\circ}$ \\
\hline ANKR & $32.76^{\circ}$ & SOFI & $23.40^{\circ}$ & SULP & $24.01^{\circ}$ \\
\hline \multirow{2}{*}{} & CRAO & $33.99^{\circ}$ & IGEO & $28.84^{\circ}$ \\
\hline
\end{tabular}




\section{Sol y viento solar}

La tormenta geomagnética de nuestro interés fue causada por una eyección de masa coronal (CME) débil producida el día 19 de abril, entre las 15:24h y las 19:30h, que tuvo dirección hacia la Tierra. Esta CME fue acompañada por una fulguración solar clasificada como C1.8.

Como refleja la Figura 2, la CME causó cambios en el viento solar. El primer efecto fue un incremento de $50 \mathrm{~km} / \mathrm{s}$ de la velocidad del viento solar acompañado de fluctuaciones de la componente $\mathrm{Bz}$ del IMF que reflejan la llegada del frente de choque asociado con la CME. Este choque se detectó el día 23 a las 3:00h. Durante los días siguientes el Sol siguió muy activo, produciéndose varias eyecciones de masa coronal que continuaron perturbando el viento solar, provocando que su velocidad aumentara hasta $700 \mathrm{~km} / \mathrm{s}$ el día 25 y que la componente $\mathrm{Bz}$ del IMF tuviera valores negativos entre las 18:00h del día 23 y las 5:00h del día 24.

Los datos recogidos por el satélite GOES, Figura 3, muestran cómo la perturbación no afectó al flujo de protones que llega a la Tierra pero sí al de electrones. Se puede observar cómo el flujo de electrones sufre dos descensos asociados a los valores negativos del Bz.

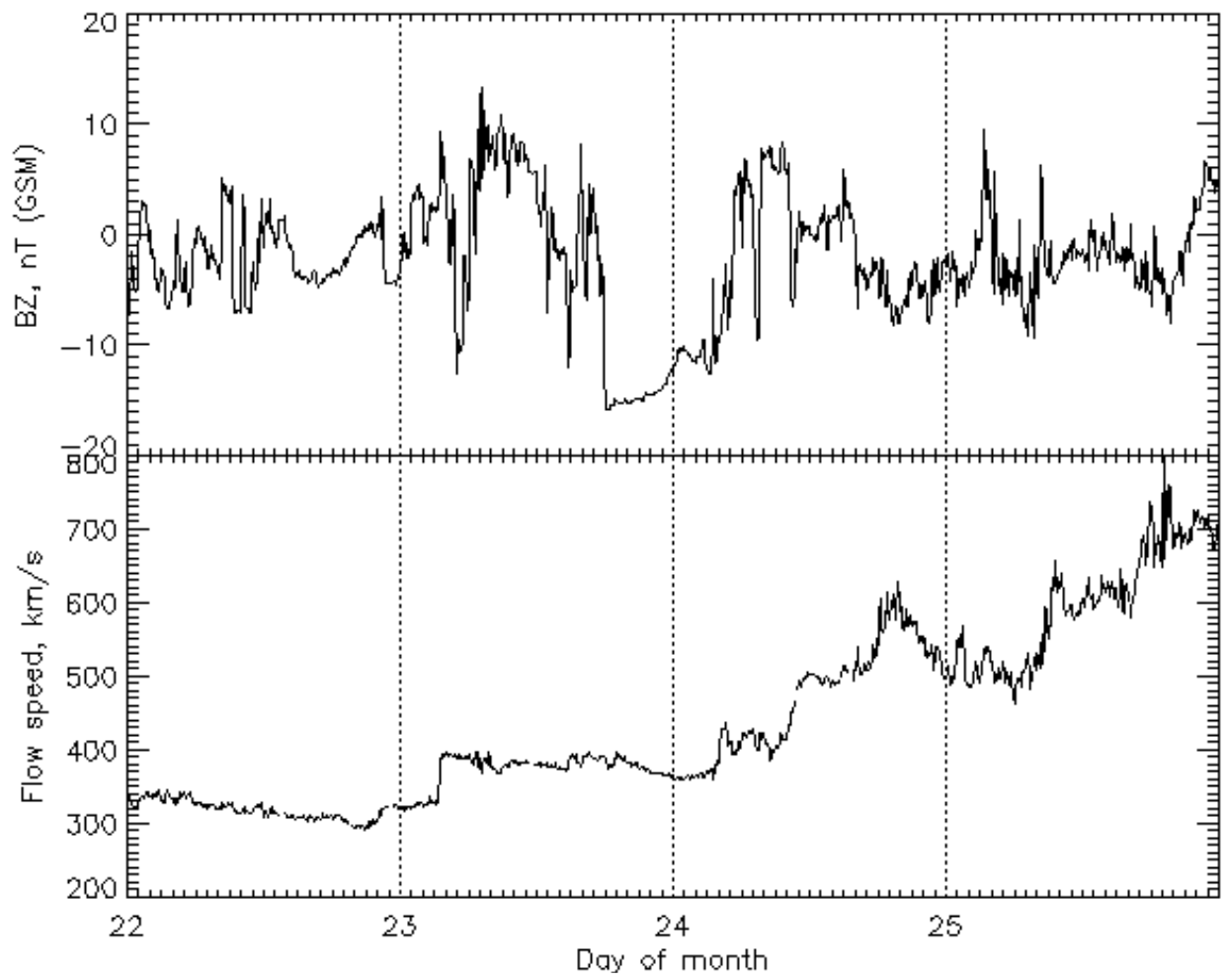

Fig. 2. Componente $\mathrm{Z}$ del IMF (arriba) y velocidad del viento solar (abajo) durante 22-25 Abril 2012 medidos con el satélite Wind (http://omniweb.gsfc.nasa.gov/). 


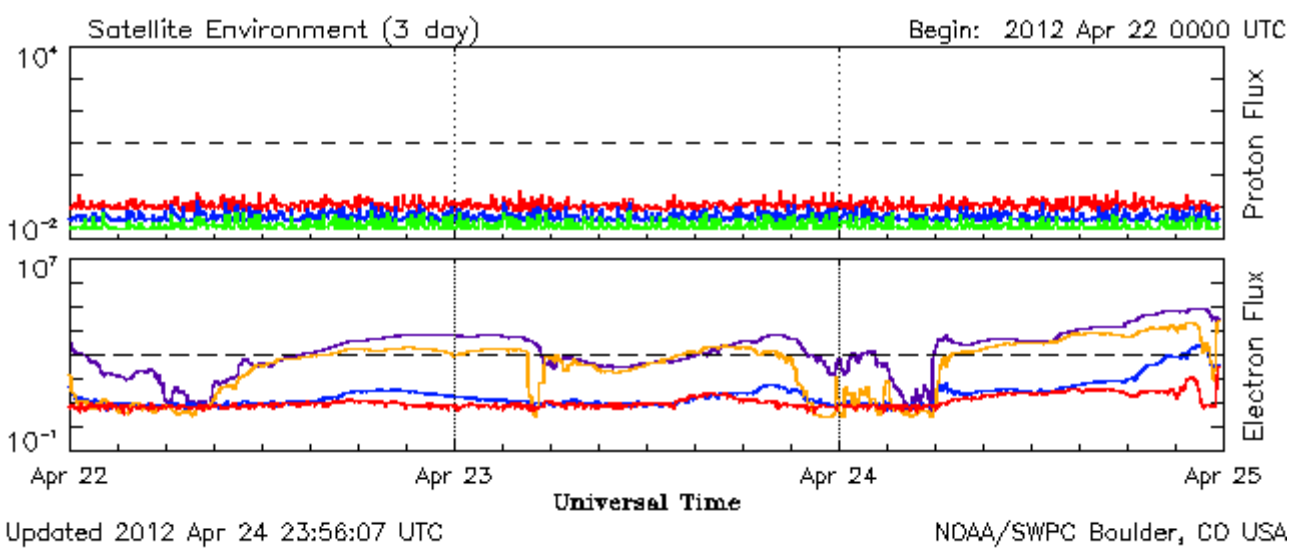

Fig. 3. Flujo de protones con distintas energías, en protones $/ \mathrm{cm}^{2} \mathrm{~s}^{-1} \mathrm{sr}^{-1}$, (arriba) y el flujo de electrones con distintas energías, en electrones $/ \mathrm{cm}^{2} \mathrm{~s}^{-1} \mathrm{sr}^{-1}$, (abajo) durante los días 22-24 de abril, de 2012, medidos con los satélite GOES 13 y 15 (http://www.swpc.noaa.gov/).

\section{Tormenta geomagnética}

Como consecuencia de la llegada a la Tierra del viento solar perturbado se inició una tormenta geomagnética el día 23 a las 4:00h que se ve reflejado en el incremento del valor de índice Dst (Figura 4) y del índice Kp (Figura 5) y en el inicio de fuertes variaciones de las componentes del campo magnético en los tres obervatorios estudiados (Figura 6). Esta pertubación del campo magnético terrestre se mantuvo hasta el día 27 cuando los valores de ambos índices regresaron a los valores típicos en condiciones tranquilas (para el índice Dst en torno a 0nT y para el índice Kp por debajo del valor 4). La máxima perturbación se alcanzó el día 24 a las 4:00h cuando el índice Dst descendió hasta -108nT, clasificando la tormenta como intensa, (González et al., 1994), y el índice Kp para el periodo entre las 3:00h y las 6:00h, alcanzó el valor de 7 de un máximo de 9.

La tormenta geomagnética tuvo 3 fases como se muestra en la Figura 4. La primera, fase inicial, se produce por la compresión de la magnetosfera debida al aumento de la presión dinámica del viento solar y origina un incremento en el índice Dst. Se inicia el día 23 hacia las 4:00h y dura 6 horas. La fase principal da comienzo a las 10:00h del día 23 y se caracteriza por una fuerte disminución del índice Dst debida al descenso de la componente horizontal del campo magnético terrestre por la inyección de plasma energizado en el anillo de corriente ecuatorial. La fase principal dura 18 horas, hasta las 4:00h del día 24 cuando se alcanza el valor mínimo mencionado anteriormente. A partir de este momento comienza la etapa de recuperación en la que el campo magnético regresa a sus condiciones en situación de equilibrio. Esta fase dura hasta el día 27, cuando concluye la tormenta geomagnética. 
En la Figura 6 se puede observar cómo la perturbación del campo magnético comienza a la misma hora en los 3 observatorios geomagnéticos del area de estudio. En los 3 casos la variación temporal del campo magnético antes de la llegada de la perturbación es prácticamente 0 , y a las $3: 30 \mathrm{~h}$ se produce una fuerte variación de $8 \mathrm{nT} / \mathrm{min}$ en los 3 observatorios seguida de pequeñas fluctuaciones que perduran el resto del día.

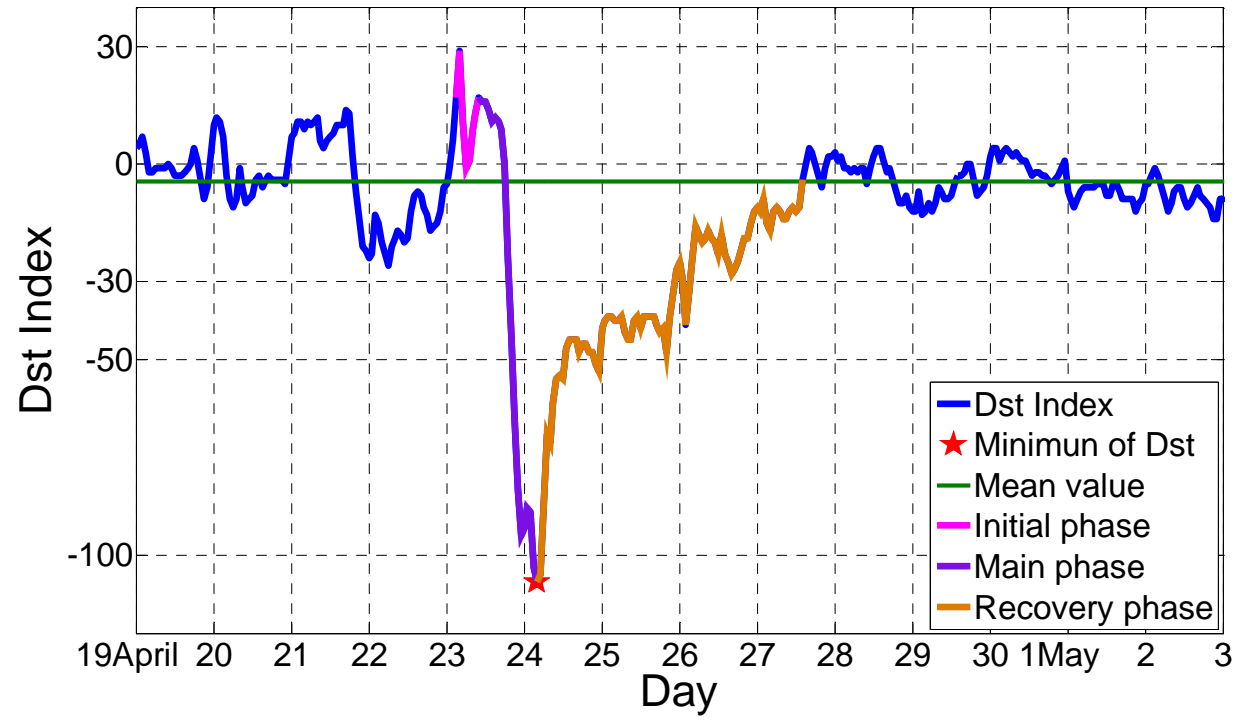

Fig. 4. Índice Dst entre los días 19 de abril y 2 de mayo.

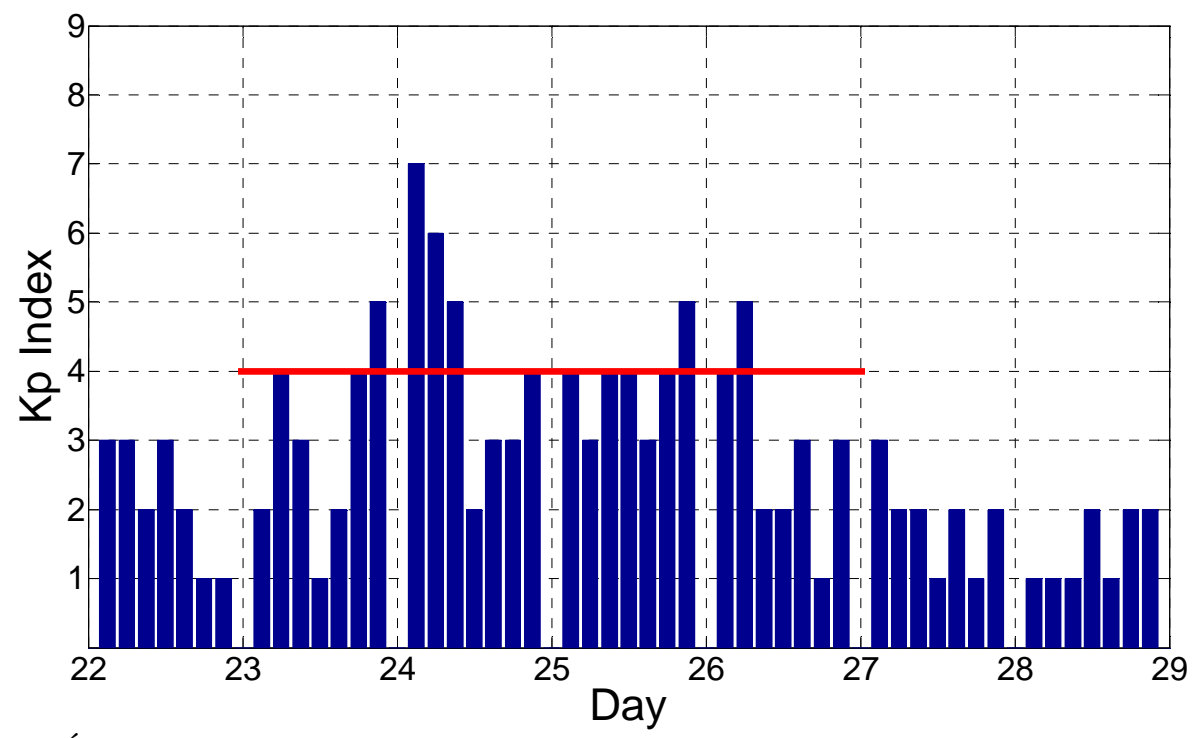

Fig. 5. Índice Kp entre los días 22 y 28 de abril. 

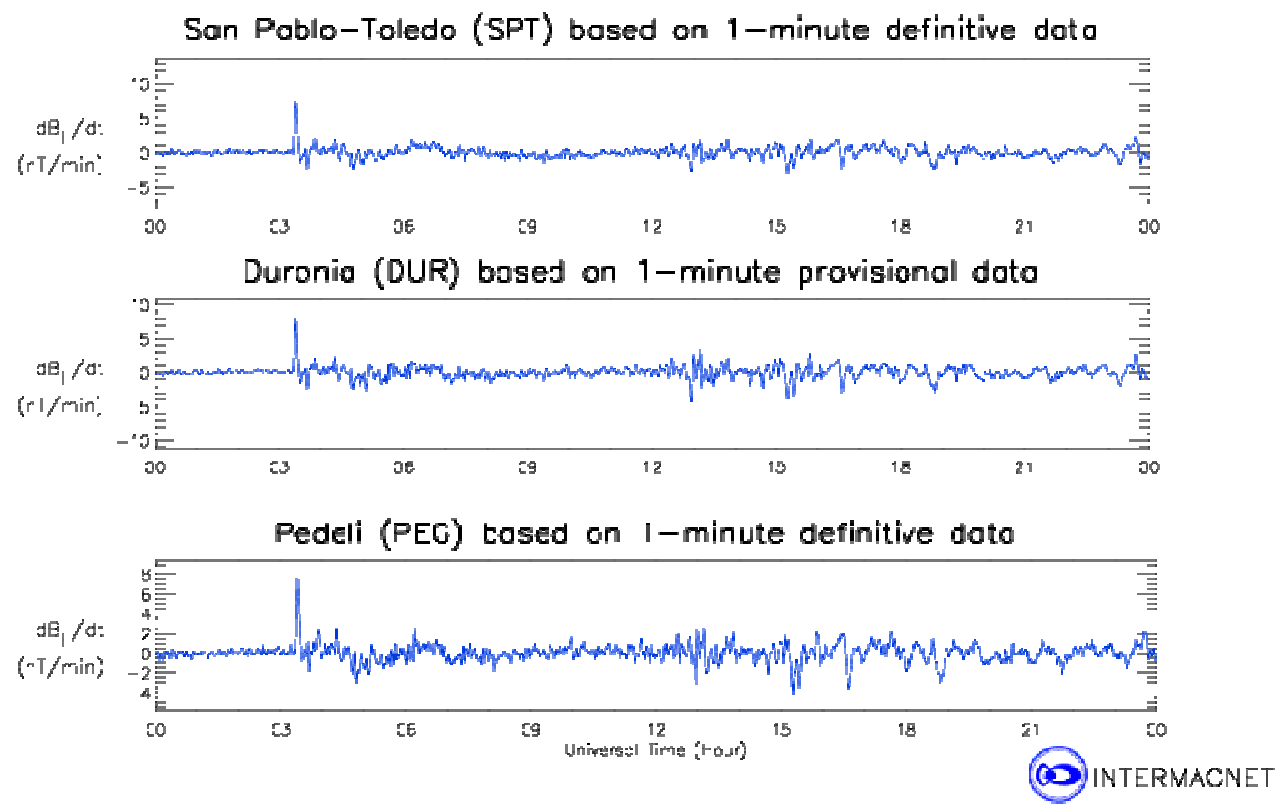

Fig. 6. Variaciones del campo magnético el día 23 de Abril en los observatorios de San Pablo de los Montes, Duronia y Pedeli (http://www.intermagnet.org/).

\section{Tormenta ionosférica}

La tormenta geomagnética analizada introdujo perturbaciones en la ionosfera sobre la región Mediterránea que dieron lugar a una tormenta ionosférica cuyas características se analizan a continuación. Estudiaremos estas tormentas a través del vTECrel. Cuando este parámetro supere el valor umbral de $\pm 40 \%$ se considerará que se produce la tormenta ionosférica.

\section{a. Efecto latitudinal}

En todos los meridianos observamos una fase negativa, Figura 7, durante, aproximadamente, todo el día 24 que coincide con el máximo de perturbación. En esta fase se observa un ascenso por encima del valor umbral de $-40 \%$ en torno a las 22:00h del día 24, para producirse un nuevo descenso a las 0:00h del día 25 que se caracteriza por ser más profundo en las latitudes más altas.

Existe otra fase común en todas las longitudes. Es una fase positiva asociada a la etapa principal de la tormenta geomagnética que solo se detecta en las latitudes más bajas de todos los meridianos. El incremento en el vTEC relativo que se produce en esta fase aumenta al descender la longitud. 
Otra fase observada en todas las longitudes es una etapa positiva tras la fase negativa principal. Sus características varían en función de la longitud aunque el efecto siempre es mayor en las estaciones situadas más al Sur. Tras la fase negativa se detectan dos ascensos del vTEC relativo, uno a las 12:00h del día 25 y otro a las 0:00h del día 26. Estos dos ascensos no llegan a superar los valores umbrales en todas las latitudes. El primer incremento solo supera el valor del $40 \%$ en los meridianos de $12^{\circ} \mathrm{E}, 16^{\circ} \mathrm{E}, 23^{\circ} \mathrm{E}$ y $34^{\circ} \mathrm{E}$, es decir, en los más orientales, mientras que el segundo se produce en los meridianos de $4^{\circ} \mathrm{W}$ y $0^{\circ} \mathrm{E}$. El meridiano de $9^{\circ} \mathrm{E}$ es el único en el que los dos ascensos superan los valores umbrales de la tormenta ionosférica.

Finalmente, el día 26 en torno a las 3:00h, se produce una nueva fase negativa que solo se detecta en las latitudes más altas en los meridianos más occidentales y que no se aprecia en el meridano de $16^{\circ} \mathrm{E}$.

\section{b. Efecto longitudinal}

En el análisis longitudinal de la tormenta ionosférica, Figura 8, también se observan las fases ya mencionadas. La primera fase positiva, correspondiente a la principal, se observa en las 3 latitudes pero en la latitud más baja, $39^{\circ} \mathrm{N}$, solo se aprecia en las estaciones situadas más al oeste.

En la fase negativa se puede ver que en la latitud más baja, Figura 8.a, la fase alcanza valores menores cuanto más al oeste está la estación, característica que no se aprecia en las otras latitudes.

La tercera fase, la correspondiente al doble ascenso, no se observa en la latitud más alta. En las estaciones en torno a $44^{\circ}$, solo se aprecia el primer ascenso mientras que en la latitud más baja observamos el primer ascenso en las estaciones más orientales, hasta $16^{\circ} \mathrm{E}$, y el segundo ascenso en las estaciones más occidentales.

En la última fase negativa, se distingue el descenso estudiado anteriormente y otro, de menor intensidad que solo está presente en algunas de las estaciones. 


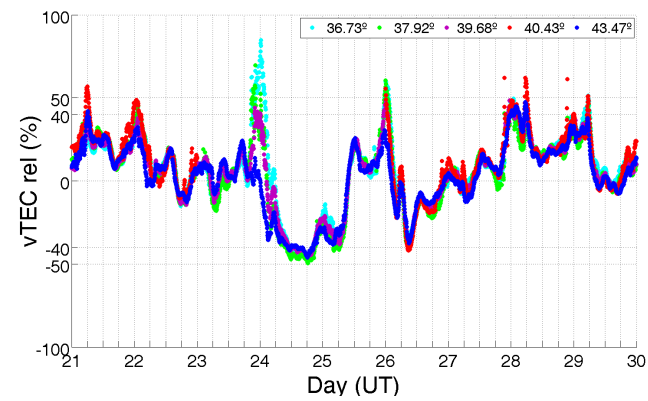

(a) Longitud $-4^{\circ}$

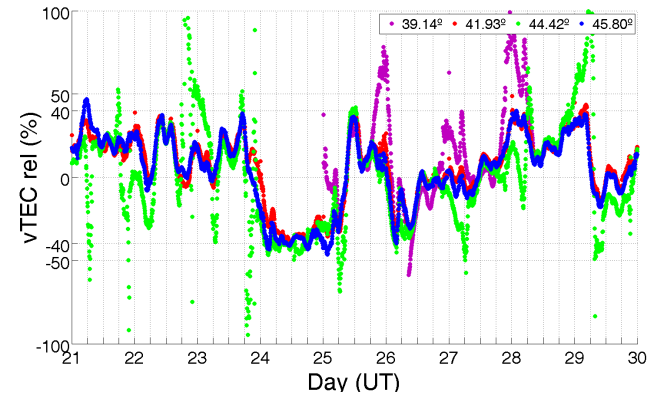

(c) Longitud $9^{\circ}$

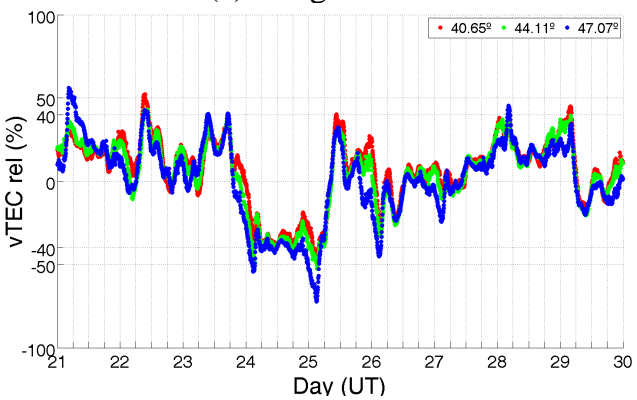

(e) Longitud $16^{\circ}$

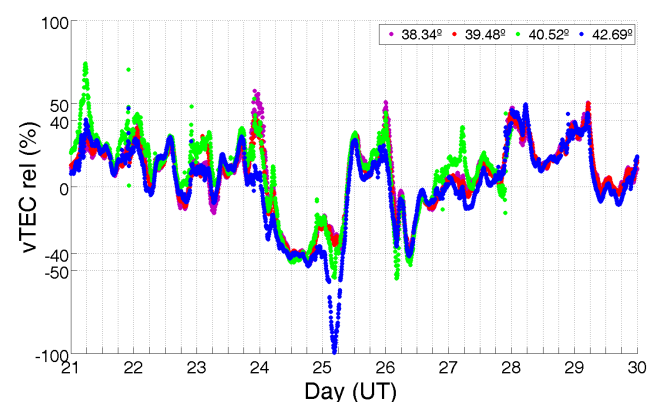

(b) Longitud $0^{\circ}$

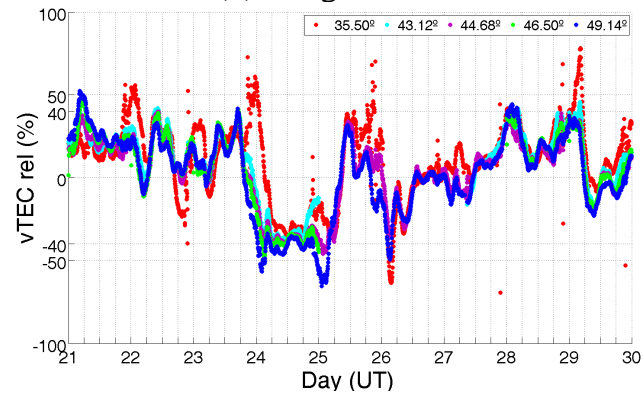

(d) Longitud $12^{\circ}$
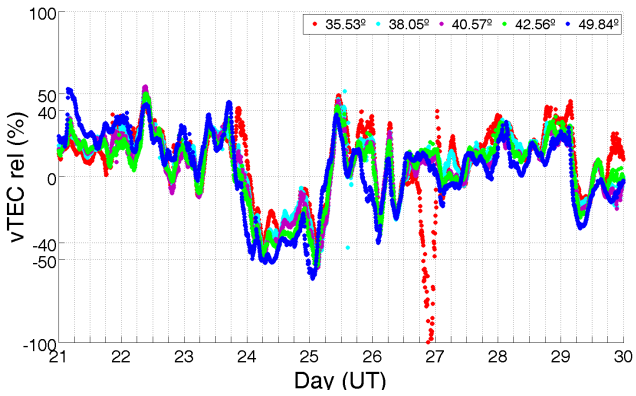

(f) Longitud $23^{\circ}$

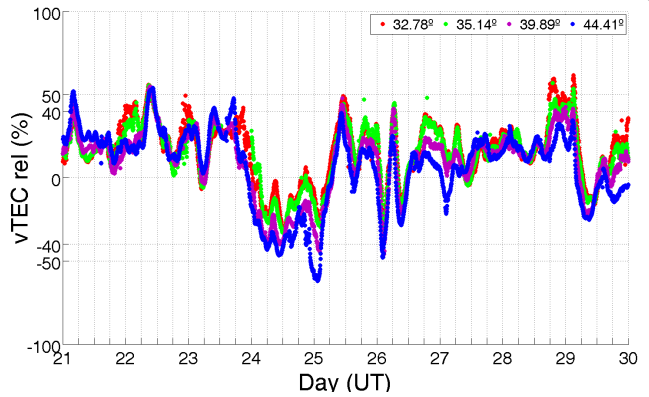

(h) Longitud $34^{\circ}$

Fig. 7. vTECrel entre los días 21 y 29 de abril. 


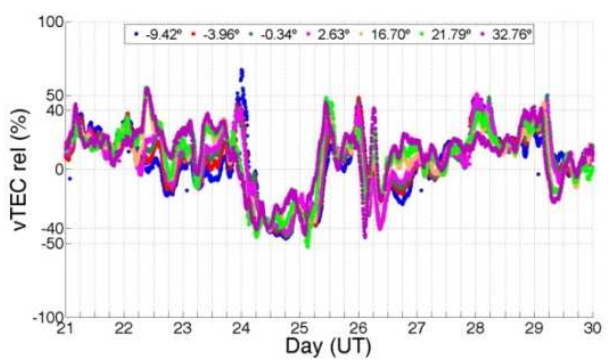

(a) Latitud $39^{\circ}$

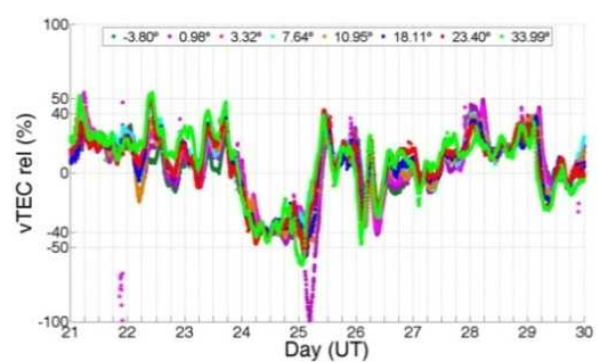

(b) Latitud $44^{\circ}$

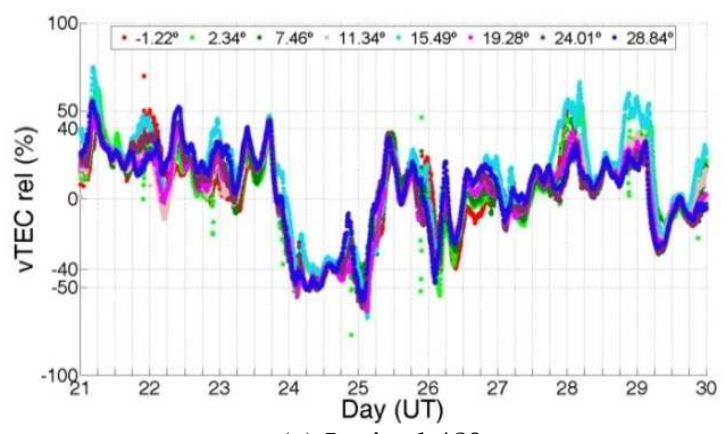

(c) Latitud $48^{\circ}$

Fig. 8. vTECrel entre los días 21 y 29 de abril.

\section{c. Efecto regional}

Para finalizar el estudio se ha obtenido el vTEC sobre toda la región y se ha analizado su comportamiento en los momentos de máxima perturbación. Para ello se ha comparado el vTEC sobre toda la región el día 24 a las 11:00h, momento de máxima perturbación negativa, y el día 25 a las 12:00, cuando se produce el mayor aumento de una de las fases positivas, con los valores del mismo parámetro en un día tranquilo previo a la tormenta y a las mismas horas.

En la Figura 9 se aprecia cómo el descenso del vTEC en la fase negativa es de unos 20TECus en toda la región. Se puede ver cómo desaparece prácticamente el gradiente horizontal de vTEC Este-Oeste y también el gradiente Norte-Sur, quedando en toda la región valores del vTEC entre 10 y 20 TECus (salvo en el Norte de Egipto donde se alcanzan los 25TECus) mientras que para un día tranquilo en la región más Sureste se encuentran valores que superan los 55 TECus y en las regiones más al Oeste en torno a los 20TECus.

Para la fase positiva, Figura 10, se ve que la influencia es menor. Se produce un incremente del vTEC en la zona Sur entre $10^{\circ} \mathrm{E}$ y $20^{\circ} \mathrm{E}$. Este incremento origina un aumento del gradiente horizontal de vTEC en dirección Norte-Sur pues los valores 
del vTEC en las latitudes más altas no aumentan tanto (unos 5TECus) como en la zona Sur (aumento de 10 TECus).
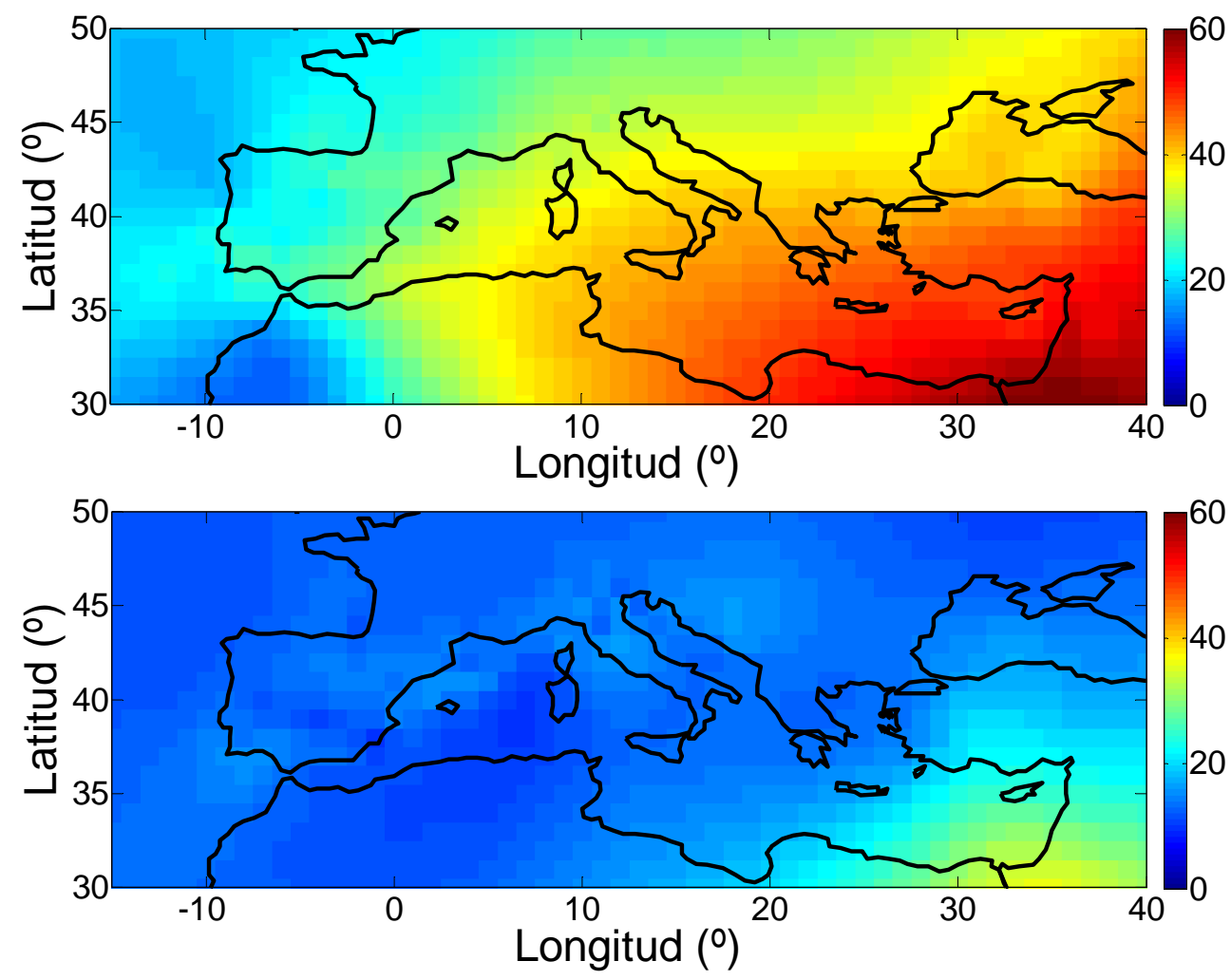

Fig. 9. vTEC sobre la región Mediterránea el día 22 (arriba) y el día 24 (abajo) a las 11:00.

\section{Conclusiones}

Se ha analizado la tormenta geomagnética del 23 de Abril de 2012 originada por la eyección de masa coronal ocurrida el 19 de Abril. La tormenta geomagnética fue intensa debido a que la perturbación del campo magnético terrestre produjo un descenso en el índice Dst hasta el valor de -108nT. La tormenta geomagnética causó una tormenta ionosférica en la región del Mediterraneo.

La tormenta ionosférica tuvo 4 fases. Una primera, positiva, asociada a la etapa principal de la tormenta geomagnética. Esta fase fue más intensa en las latitudes más bajas y en la zona más occidental del área estudiada. La fase más importante de la tormenta ionosférica es la segunda, de carácter negativo y con una duración aproximada de un día, que comenzó en el momento de máxima perturbación, al final de la fase principal de la tormenta geomagnética, y permaneció durante la 
primera parte de la fase de recuperación. En esta fase aparece un descenso final del vTEC mayor en las latitudes más altas y sin ninguna dependencia en la longitud. Tras la fase negativa aparece una nueva fase positiva. El incremento del vTEC en esta fase en estaciones más al este del meridiano $9^{\circ} \mathrm{E}$ tiene lugar el día 25 a las 12:00h mientras que en las estaciones al oeste de este meridiano se produce el día 26 a las 0:00h. En el meridiano de $9^{\circ} \mathrm{E}$ se produce incremento en las dos horas señaladas pero el segundo solo aparece en las latitudes más bajas. Finalmente se genera una fase negativa que en las zonas más orientales se detecta en todas las latitudes mientras que en las occidentales solo en las latitudes más altas.

Las dos primeras fases descritas corresponden a las dos fases caracteristicas de las tormentas ionosféricas en latitudes medias. La primera, la fase positiva, se debe al incremento de los vientos ecuatoriales y se intensifica por el efecto de la puesta del Sol. La fase principal es la fase negativa que tiene mayor duración y está causada por los cambios en el ratio $\mathrm{O} / \mathrm{N}_{2}$ (Mendillo, 2006).

Respecto a las características de la tormenta sobre toda la región se puede señalar que durante la fase negativa se produce un descenso generalizado del vTEC que lleva a que los valores del vTEC sean similares en toda la región y, por tanto, desaparezcan tanto los gradientes horizontales Este-Oeste como los gradiente Norte-Sur. Para las fases positivas se aprecia un incremento del gradiente Sur-Norte de vTEC.

\section{Agradecimientos}

Este trabajo forma parte de la actividad investigadora del "Grupo de Estudios Ionosféricos y Técnicas de Posicionamiento Satelital (GNSS)" (Grupo: 910596), subvencionado por la Universidad Complutense de Madrid y la Fundación Mapfre. Los autores agradecen a L. Ciraolo por facilitar el algoritmo que permite procesar los datos. También quieren agradecer a las distintas instituciones y servicios que publican los datos (Goddard Space Flight Center Space Physics Data Facility, World Data Center for Geomagnetism de Kyoto, Intermagnet), a las redes de estaciones permanentes GNSS (EUREF Permanent Network (EPN) e International GNSS Service, IGS) por permitir el uso de los datos y a la Federal Agency for Cartography and Geodesy de Alemania, por facilitar el acceso a los archivos RINEX.

También se desea agradecer a Consuelo Cid su trabajo de revisión que ha ayudado a mejorar el artículo. 

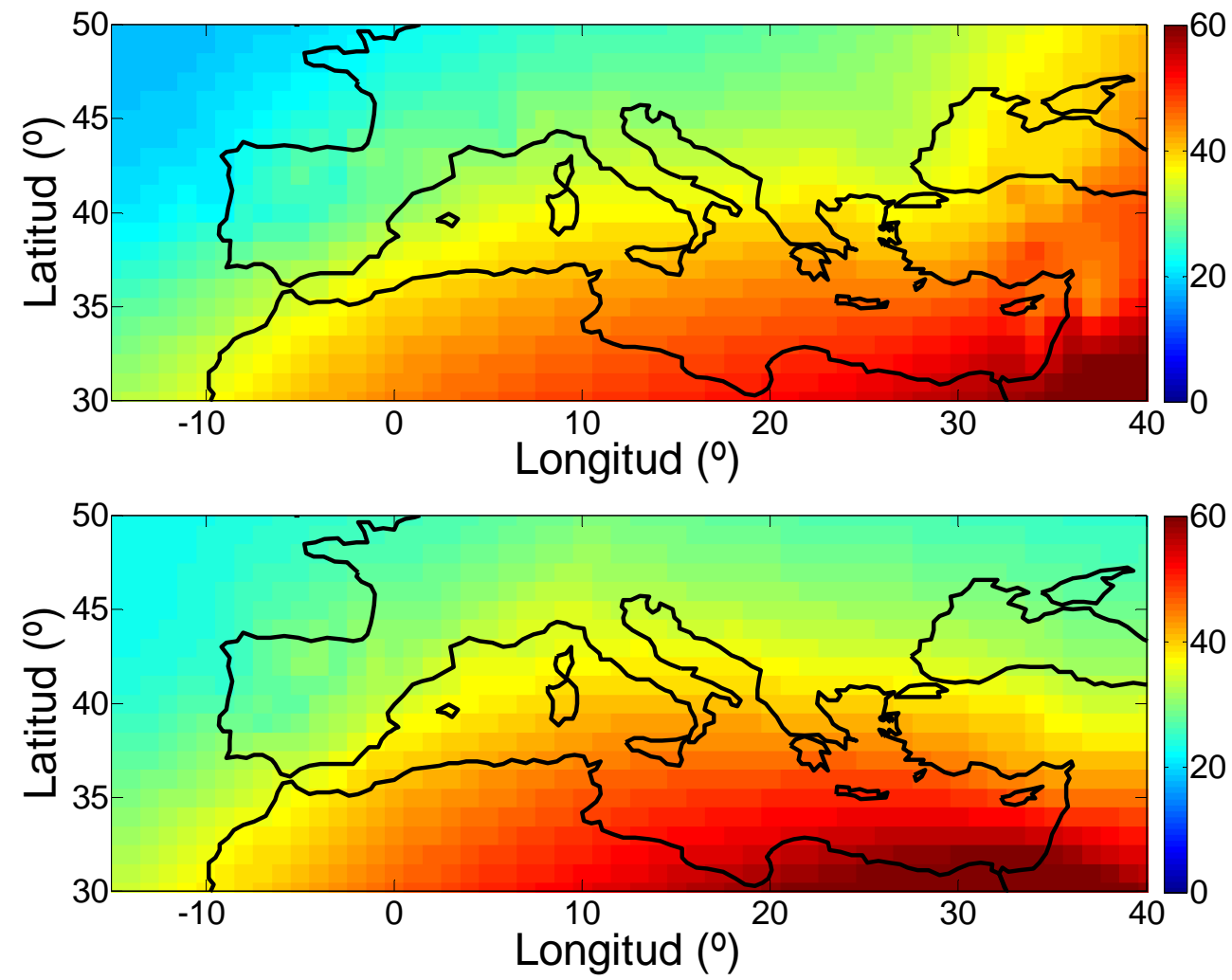

Fig. 10. vTEC sobre la región Mediterránea el día 22 (arriba) y el día 25 (abajo) a las 12:00.

\section{Referencias Bibliográficas}

BASU, S.U., BASU, S., MAKELA, J. J., MACKENZIE, E., DOHERTY, P., WRIGHT, J. W., RICH, F., KESKINEN, M. J., SHEEHAN, R. E., COSTER, A. J. (2008). Large magnetic storm-induced nighttime ionospheric flows at midlatitudes and their impacts on GPS-based navigation systems. J. Geophys. Res., 113, A00A06, doi: 0.1029/2008JA013076.

BERGEOT, N., BRUYNINX, C., DEFRAIGNE, P., PIREAUX, S., LEGRAND, J., POTTIAUX, E., BAIRE, Q. (2011). Impact of the Halloween 2003 ionospheric storm on kinematic GPS positioning in Europe. GPS Solutions, 15,171-180.

BUONSANTO, M.J. (1999). Ionospheric storms- A review. Space Science Reviews, $88,563-601$.

CIRAOLO, L. (2012). Ionospheric Total Electron Content (TEC) from Global Positioning System. Comunicación personal.

GONZÁLEZ, W.D., JOSELYN, J. A., KAMIDE, Y., KROEHL, H. W., ROSTOKER, G., TSURUTANI, B. T., VASYLIUNAS, V. M. (1994). What is a 
Geomagnetic Storm?. J. Geophys. Res., 99, 5771-5792, doi:10.1029/93JA02867.

HUANG, C.-S., FOSTER, J.C., GONCHARENKO, L.P., ERICKSON, L.P., RIDEOUT, W., COSTER, A.J. (2005). A strong positive phase o ionospheric storm observed by the Millstone Hill incoherent scatter radar and global GPS network., J. Geophys. Res., 110, A06303, doi: 10.1029/2004JA010865.

KELLY, M.N., (2009). The Earth's Ionosphere. Plasma Physics and Electrodynamics. Cornell University College of Engineering School of Electrical and Computer Engineering, Ithaca, NY.

KOMJATHY, A., SPARKS, L., MANUCCI, A., COSTER, A. J. (2005). The ionospheric impact of the October 2003 storm event on Wide Area Augmentation System. GPS Solutions, 9,41-50.

MENDILLO, M. (2006). Storms in the ionopshere: patterns and processes fot total electron content. Rev. Gephys., 44 RG4001, doi:10.1029/2005RG000193.

PARKISNSON, W.D. (1983). Introduction to Geomagnetism. Scottish Acdeminc Press.

SPOOGLI, L., ALFONSI, L., DE FRANCESCHI, G., ROMANO, V., AQUINO, M. H., DOBSON, A. (2009). Climatology of GPS ionospheric scintillations over high and mid-latitude European regions. Ann. Geophys., 27, 3429-3437.

WEBSTER, R., OLIVER, M. (2001). Geostatistics for Environmental Scientists. Wiley Publishing, New York http://wdc.kugi.kyoto-u.ac.jp/index.html http://igscb.jpl.nasa.gov http://www.epncb.oma.be/ http://omniweb.gsfc.nasa.gov/ http://www.swpc.noaa.gov/ http://www.intermagnet.org/ 